\section{Cross-transmission rates of enterococcal isolates among newborns in a neonatal intensive care unit}

\author{
Henning Böhme, 1 Chitra Königsmark,2 \\ Ingo Klare, ${ }^{3}$ Melanie Zischka, ${ }^{3}$ \\ Guido Werner ${ }^{3}$ \\ 1Children's Hospital, ${ }^{2}$ Centre for \\ Laboratory Diagnostics, Harz-Klinikum \\ Wernigerode-Blankenburg $\mathrm{GmbH}$; \\ ${ }^{3}$ Robert Koch-Institute, Wernigerode \\ Branch, Wernigerode, Germany
}

\begin{abstract}
Enterococci are important pathogens causing nosocomial infections and patients at risk include also premature babies requiring intensive care treatment. Our aim was to assess occurrence and cross transmission rates of enterococci among neonatal patients of a hospital ward during a two months period. Rectal and skin samples were taken between day one and 60 of infants' age. Colonization with various potentially pathogenic bacteria was correlated with developing a subsequent infection. Enterococcal isolates were identified by colony morphology. The bacterial species was assessed and antibiotic susceptibilities were determined. A molecular analysis of 20 investigated enterococcal isolates revealed prevalence of commensal strain types; hospitalassociated strain types or multi-resistant variants were absent. Cross transmission of $E$. faecium and $E$. faecalis isolates among neonatal patients attending the intensive crare unit at the same time was demonstrable. Introduction of hospital-associated, multi-resistant variants into this special setting has to be avoided to reduce the risk of subsequent infections.
\end{abstract}

\section{Introduction}

Rates of enterococcal infections among patients in intensive care increased dramatically in the last two decades worldwide. ${ }^{1}$ Enterococcal isolates (Enterococcus faecalis and $E$. faecium) are ranked as the second most important nosocomial pathogen in intensive crare unit (ICU) acquired bloodstream infections in Europe. ${ }^{2}$ Infections occur in severely ill and immuno-compromised patients revealing also premature babies and newborns at risk for acquiring enterococcal infections. . $^{3,4}$ Transmission rates of enterococcal isolates are high among nosocomial patients, even higher than for $S$. aureus and E. coli. ${ }^{5}$ Increased importance of enterococci especially $E$. faecium as a nosocomial pathogen is linked to a preferred prevalence of hospital-associated strain types that possess acquired antibiotic resistance properties, specific molecular markers and clonal types identifiable by molecular typing techniques such as Multi-locus sequence typing (MLST). ${ }^{6}$ On the other hand, commensal clonal types of $E$. faecalis and $E$. faecium exhibit important constituents of the healthy human intestinal flora and are among the first and early colonizers of the infants' intestines. ${ }^{7}$ In the present study we investigated rectal (and skin) colonization, cross-transmission rates and clonal types of enterococci among newborns of a neonatal ICU of a German hospital over a period of two months.

\section{Brief Report}

From April to May 2006, 20 patients attending a German neonatal ICU were screened for bacterial rectal and skin colonizations. All newborns were admitted to a separate ward and were kept aside from other pediatric patients. Samples were taken in relation to clinical signs of infection of the babies (fever, etc.) or when mothers had risk factors or infections (amnion infection, vaginal group B streptococcus colonization, etc). Potentially pathogenic bacteria such as Staphylococcus aureus, coagulase-negative staphylococci, Enterobacteriaceae and Enterococcus spp. were assessed. Five patients were rectally screened consecutively, two and three times, respectively (Table 1). Rectal and skin samples were primarily screened on two non-selective media; Brilliance UTI Clarity agar (Oxoid/Thermo Fischer Scientific, Wesel, Germany) and Trypticase Soy-Agar + 5\% Sheep Blood (bioMerieux, Nuertingen, Germany). Morphologically different isolates per sample were further identified by Vitek $^{\circledR} 2$ (bioMerieux, Nürtingen, D). All but three enterococcal isolates were available for a subsequent analysis. Antibiotic susceptibilities for enterococci were initially determined by VITEK$^{\circledR} 2$ (bioMérieux, according to CLSI criteria and later confirmed by an in house microbroth dilution method. ${ }^{8}$ Epidemiological markers to differentiate hospital-associated strains of $E$. faecium from colonizing variants were determined by PCR as described recently (esp, $\left.h y l_{E f m}, I S 16\right) . .9,10$ To investigate clonal relatedness of strains macrorestriction analysis in Pulsed-Field Gel Electrophoresis was performed as described ${ }^{9}$ and subsequent analysis was done using a Dice coefficient and UPGMA clustering (BioNumercis v. 5.1; Applied Maths, Belgium). Multi-locus sequence typing (MLST) was performed to differentiate between commensal and hospital-associated
Correspondence: Guido Werner, FG 13 Nosocomial Infections, Robert Koch Institute, Wernigerode Branch, Burgstrasse 37 D-38855 Wernigerode, Germany.

Tel: +49.03943679210 - Fax: +49.03943679 $207 / 317$

E-mail: wernerg@rki.de

Key words: enterococcus, PFGE, nosocomial spread.

Acknowledgments: we acknowledge excellent laboratory assistance by Mrs. Carola Fleige and Mrs. Uta Geringer. MZ is supported by a PhD fellowship grant from the Robert Koch Institute.

Contributions: HB, GW designed the study; HB performed the study; CK did the primary diagnostics; MZ, IK, GW did the phenotypic confirmatory diagnostics and molecular analysis; GW, HB wrote the manuscript.

Conflicts of interest: all authors declare that there are no conflicts of interest.

Received for publication: 19 August 2011.

Revision received: 1 February 2012.

Accepted for publication: 2 February 2012.

This work is licensed under a Creative Commons Attribution NonCommercial 3.0 License (CC BYNC 3.0).

(C) Copyright H. Böhme et al., 2012

Licensee PAGEPress, Italy

Pediatric Reports 2012; 4:e15

doi:10.4081/pr.2012.e15

strain types as given elsewhere (http://efaecium.mlst.net/ and http://efaecalis.mlst.net).

\section{Results}

Of the 20 screened neonatal patients only two samples did not grow bacteria at all (of the genera and species described above; Table 1). Ten neonatal patients showed signs of an infection and received immediate antibiotic treatment (Table 1). Screening samples of 18 patients grew enterococci and from 14 children isolates were accessible. From two patients consecutive isolates were analyzed (Figure 1). Altogether eight $E$. faecalis and eight $E$. faeci$u m$ isolates were available for a molecular analysis. Antibiotic susceptibility testing revealed a very small spectrum of acquired resistances; mainly erythromycin (E. faecium) and tetracycline ( $E$. faecalis; Figure 1) representing widespread acquired resistance characteristics also prevalent among animal, environmental and human colonizing, commensal enterococcal strains. Molecular markers of hospital-associated E. faecium strains (esp, 


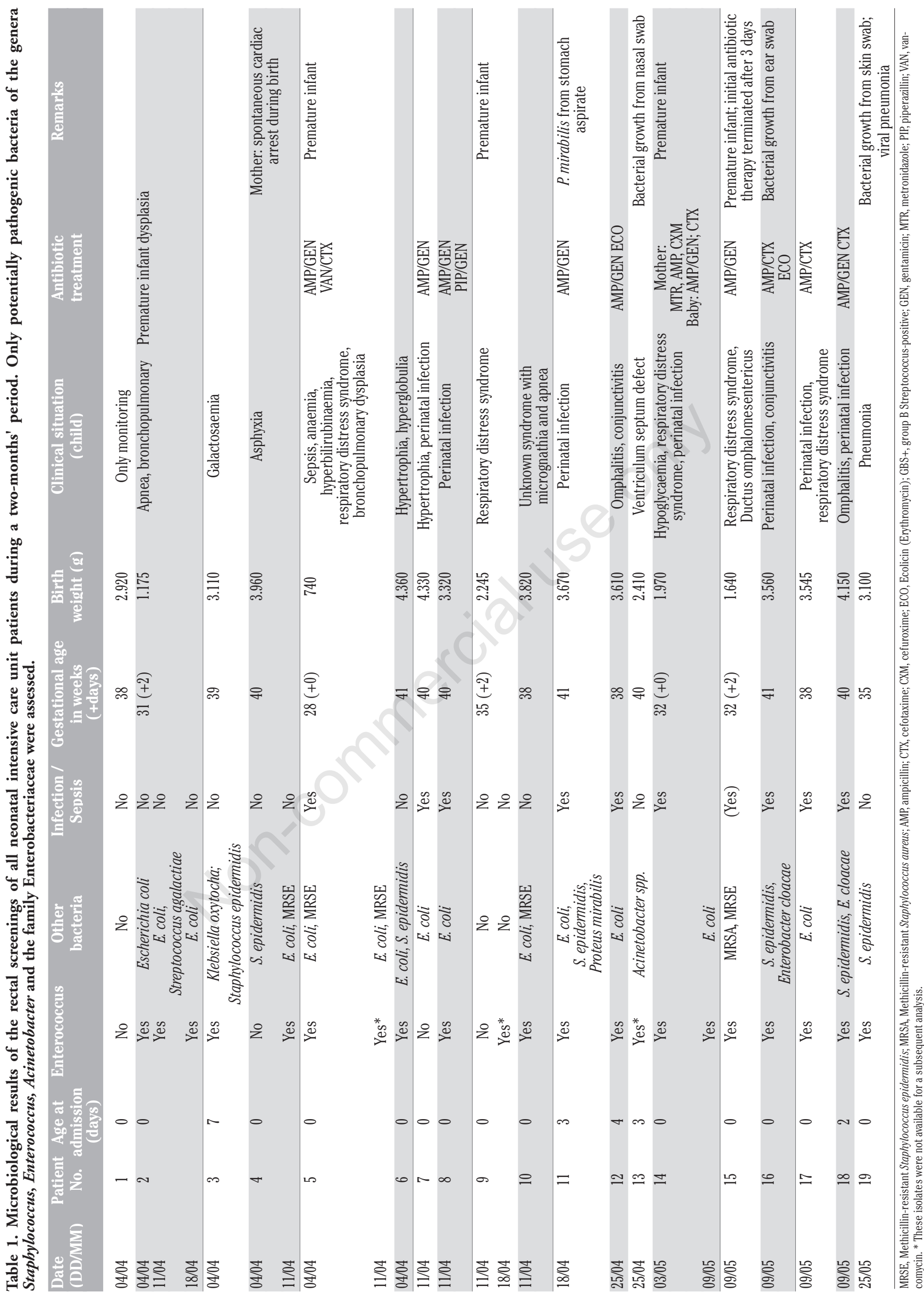




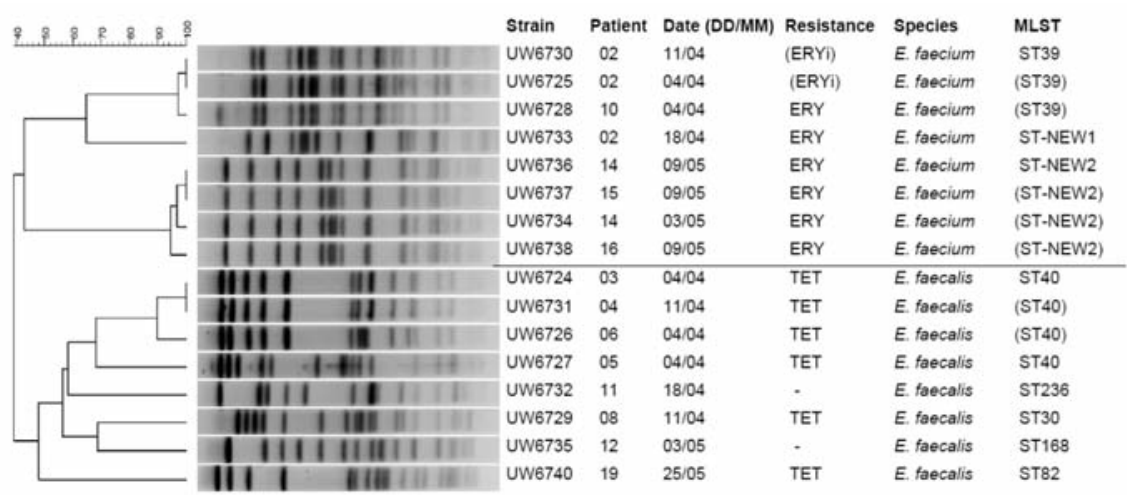

Figure 1. SmaI macrorestriction patterns in Pulsed-Field Gel Electrophoresis (PFGE) of all enterococcal isolates colonizing neonatal patients. Clusters of related isolates from samples of different neonates suggest horizontal transmission between patients (clonal spread), for instance, isolates $E$. faecalis UW6724 [Patient no. 3], UW6731 [Patient no. 4], and UW6726 [Patient no. 6]. MLST types in parentheses are predicted according to an identical PFGE profile. Profiles ST-NEW1 and ST-NEW2 are new MLST types due to new $(\mathrm{N})$ allele sequences which have the following allelic profiles: ST-NEW 1 [N-8-8-N6-27-6]; ST-NEW2 [25-8-N-17-10-N-6] (see also http://efaecium.mlst.net/ and http://efaecalis.mlst.net/). ERY(i), erythromycin (intermediate); TET, tetracycline; MLST, Multi-locus sequence typing; ST, sequence type (of MLST); n.d., not determined.

$h y l_{E f m}$ IS16) were absent in the eight $E$. faecium isolates. MLST results confirmed prevalence of commensal $E$. faecium strain types among the investigated patients (Figure 1). Four isolates of $E$. faecalis revealed MLST strain type ST40 known to be highly prevalent among various ecological sources and which is also isolated from severe invasive infections like endocarditis and sepsis 11 and from cases of bovine mastitis (Zischka and Werner, unpublished data). However, hospital-associated strain types belonging to clonal complexes CC2 and CC9 were not identified among the $E$. faecalis isolates. Cross transmission between the newborn babies attending the neonatal ICU at the same time period was demonstrated for $E$. faecium and E. faecalis isolates as well as ongoing colonization with identical strain types in single neonates (Figure 1).

\section{Conclusions}

A microbiological and molecular analysis of the enterococcal isolates from stool colonizations in newborn infants of a neonatal ICU revealed prevalence of colonizing strain types. Hospital-associated strain types or multiresistant variants were not identified. Nevertheless, also colonizing strain types could cause endogenous enterococcal infections; however, the pathogens causing the described invasive infections could not been identified here. Cross transmission of enterococcal isolates among newborns attending the neonatal ICU at the same time was shown. To elucidate the source of these early colonizing isolates, an independent and comprehensive follow-up study including stool sampling and subsequent analyses from babies' mothers and medical staff is planned.

\section{References}

1. Arias CA, Murray BE. Antibiotic-resistant bugs in the 21st century--a clinical superchallenge. N Engl J Med 2009;360:439-43.
2. Fifteen most frequently isolated microorganisms in ICU-acquired bloodstream infections by country, results from the HAI-HELICS projects, 2008. In: Annual epidemiological report on communicable diseases in Europe in 2008. Available from: www.ecdc.europa.eu

3. Haas EJ, Zaoutis TE, Prasad P, et al. Risk factors and outcomes for vancomycinresistant Enterococcus bloodstream infection in children. Infect Control Hosp Epidemiol 2010;31:1038-42.

4. Faust K, Goepel W, Herting E, Haertel C. [Sepsis in very low weight birth infants.] Chemother J 2010;20:1-8. [Article in German]

5. Barwolff S, Grundmann H, Schwab F, et al. [Incidence of transmission of pathogens in intensive care units. Results of the SIR 3 study]. Anaesthesist 2005;54:560-6. [Article in German]

6. Leavis HL, Bonten MJ, Willems RJ. Identification of high-risk enterococcal clonal complexes: global dispersion and antibiotic resistance. Curr Opin Microbiol 2006;9:454-60.

7. Fanaro S, Chierici R, Guerrini P, Vigi V. Intestinal microflora in early infancy: composition and development. Acta Paeditr Suppl 2003;441:48-55.

8. CLSI. Performance Standards for Antimicrobial Susceptibility Testing, 16th Informational Supplement, M100-S16, 2006;26(3):52-55. Available from: www.microbiolab -bg.com/CLSI.pdf

9. Werner G, Fleige C, Ewert B, et al. Highlevel ciprofloxacin resistance among hospital-adapted Enterococcus faecium (CC17). Int J Antimicrob Agents 2010;35: 119-25.

10. Werner G, Fleige C, Geringer U, et al. IS element IS16 as a molecular screening tool to identify hospital-adapted strains of $\mathrm{E}$. faecium. BMC Inf Dis 2011;11:80.

11. McBride SM, Fischetti VA, Leblanc DJ, et al. Genetic diversity among Enterococcus faecalis. PLoS One 2007;2:e582. 